\title{
Integrating microbial and host transcriptomics to characterize asthma-associated microbial communities
}

\author{
Eduardo Castro-Nallar ${ }^{1,5^{*}}$, Ying Shen ${ }^{2}$, Robert J. Freishtat ${ }^{3}$, Marcos Pérez-Losada ${ }^{1,3,4}$, Solaiappan Manimaran², \\ Gang Liü ${ }^{2}$ W. Evan Johnson ${ }^{2}$ and Keith A. Crandall ${ }^{{ }^{*}}$
}

\begin{abstract}
Background: The relationships between infections in early life and asthma are not completely understood. Likewise, the clinical relevance of microbial communities present in the respiratory tract is only partially known. A number of microbiome studies analyzing respiratory tract samples have found increased proportions of gamma-Proteobacteria including Haemophilus influenzae, Moraxella catarrhalis, and Firmicutes such as Streptococcus pneumoniae. The aim of this study was to present a new approach that combines RNA microbial identification with host gene expression to characterize and validate metagenomic taxonomic profiling in individuals with asthma.
\end{abstract}

Methods: Using whole metagenomic shotgun RNA sequencing, we characterized and compared the microbial communities of individuals, children and adolescents, with asthma and controls. The resulting data were analyzed by partitioning human and microbial reads. Microbial reads were then used to characterize the microbial diversity of each patient, and potential differences between asthmatic and healthy groups. Human reads were used to assess the expression of known genes involved in the host immune response to specific pathogens and detect potential differences between those with asthma and controls.

Results: Microbial communities in the nasal cavities of children differed significantly between asthmatics and controls. After read count normalization, some bacterial species were significantly overrepresented in asthma patients (Wald test, p-value $<0.05$ ), including Escherichia coli and Psychrobacter. Among these, Moraxella catarrhalis exhibited $\sim 14$-fold over abundance in asthmatics versus controls. Differential host gene expression analysis confirms that the presence of Moraxella catarrhalis is associated to a specific M. catarrhalis core gene signature expressed by the host.

Conclusions: For the first time, we show the power of combining RNA taxonomic profiling and host gene expression signatures for microbial identification. Our approach not only identifies microbes from metagenomic data, but also adds support to these inferences by determining if the host is mounting a response against specific infectious agents. In particular, we show that M. catarrhalis is abundant in asthma patients but not in controls, and that its presence is associated with a specific host gene expression signature.

\section{Background}

The human microbiome [1] plays a key role in a variety of human health issues from obesity [2] to respiratory disease [3]. As we advance our understanding of the diversity of microbiomes across geography, time, individuals, and tissues within individuals, we become better positioned to take advantage of this growing wealth of information on

\footnotetext{
* Correspondence: eduardo.castro@unab.cl; kcrandall@gwu.edu

${ }^{1}$ Computational Biology Institute, George Washington University, Ashburn,

VA 20147, USA

Full list of author information is available at the end of the article
}

the diversity of the human microbiome and how that diversity changes with infection and disease. Early studies capitalized on $16 \mathrm{~S}$ ribosomal data for bacterial characterizations because of the ease of data collection and the robust and growing reference databases. However, with the declining costs of high-throughput sequencing (HTS) and the limitations of single gene inferences, microbiome studies are increasingly relying on shotgun metagenomics to obtain more complete profiles of microbial communities. An immediate concern is the sheer volume of data generated by the metagenomics approach, which presents novel 
challenges for efficient data handling and analysis. These challenges are especially acute when attempting to identify relevant microbes for suspected infections - trying to differentiate microbes relevant to the host from microbes that do not elicit a response from the host is a daunting task. A variety of techniques have been developed to isolate potential pathogens for HTS targets using molecular biological approaches. However, this limits the inferences with respect to the host response. Other approaches, for instance, dual RNA-Seq has been recently suggested as a promising approach to assess differential gene expression in both the pathogen and the host from the same sample [4].

Microbiome studies of human disease typically focus on correlates between microbial composition and disease phenotype at single or multiple points in time. However, this poses significant problems when it comes to elucidating potentially causal relationships. The lingering question is whether disease results in a certain microbiome or whether this microbiome is the underlying cause of the disease. Prospective studies have attempted to establish causality relationships by monitoring microbial populations before and after the onset of disease. In the case of asthma, prospective studies have identified $H$. influenzae, M. catarrhalis, and S. pneumoniae colonization as potential risks factors [5]. Colonization with these three bacterial species has also been linked to the development of severe pulmonary infections, however this association has only been seen in children that did not develop asthma [6]. In addition, the lung microbiome project and others have proposed a core pulmonary microbiome of healthy individuals that includes genera such as Streptococcus, Haemophilus, and Pseudomonas (same order as M. catarrhalis), which casts a shadow on elucidating the role of such bacterial species in asthma [7-9].

Here, we present a computational strategy - combining RNA microbial identification and host differential gene expression signatures - to identify pathogens associated with asthma in children and supported by differences in the patients' responses to infection (host immune responserelated gene expression signatures). We tested whether microbial composition (viral, fungal, and bacterial) is significantly different between asthma individuals and controls, and whether differentially abundant microbes with available host gene signatures are associated to genes related to the immune response by the host.

\section{Methods}

\section{Sample collection}

Participants were part of the AsthMaP (Asthma Severity Modifying Polymorphisms) Project (Table 1). The AsthMaP Project was a single-center observational study of asthma. AsthMaP participants ranged between the ages of 6 and 20 years, with physician-diagnosed asthma for at least one year prior to the time of recruitment from the emergency
Table 1 Demographic data from asthma and control subjects

\begin{tabular}{|c|c|c|}
\hline \multirow[t]{2}{*}{ Variable } & Asthma $(n=8)$ & Control $(n=6)$ \\
\hline & Mean $(95 \% \mathrm{Cl})$ & Mean $(95 \% \mathrm{Cl})$ \\
\hline Gender, \% male & 75 & 83 \\
\hline Age, years, median (range) & $11(6,17)$ & $15(10,20)$ \\
\hline $\begin{array}{l}\mathrm{FEV}_{1} \text { (\% change with } \\
\text { bronchodilator), median (range) }\end{array}$ & $3.5(-13,10)$ & N/A \\
\hline $\begin{array}{l}\text { Post-bronchodilator } \mathrm{FEV}_{1} \\
\text { (\% predicted), median (range) }\end{array}$ & $97(62,107)$ & N/A \\
\hline $\begin{array}{l}\mathrm{FEF}_{25-75}(\% \text { predicted), } \\
\text { median (range) }\end{array}$ & $83(28,112)$ & N/A \\
\hline $\begin{array}{l}\text { Post-bronchodilator } \mathrm{FEF}_{25-75} \\
\text { (\% predicted), median (range) }\end{array}$ & $93(37,110)$ & N/A \\
\hline Serum IgE, IU/mL, median (range) & $247(60,1706)$ & N/A \\
\hline Blood eosinophils, \%, median (range) & $6(2,14)$ & N/A \\
\hline ACT score, median (range) & $23(17,23)$ & N/A \\
\hline
\end{tabular}

FEV 1 Forced Expiration Volume, FEF Forced Expiratory Flow, ACT Asthma Control Test. $N / A=$ information not available

department, inpatient units and outpatient clinics. Individuals who reported a medical history of chronic or complex cardiorespiratory disease were ineligible. Control subjects were confirmed not to have asthma through negative response to multiple survey questions for asthma diagnosis, symptoms, medication use, and healthcare utilization. Specific AsthMaP methodology has been published elsewhere [10-13]. Our Institutional Review Board approved this study and parents and participants gave consent/assent.

Nasal epithelial cells were collected from 8 children and adolescents with asthma and 6 healthy controls by brushing the medial aspect of the inferior turbinate of each nare using a cytology brush. Nasal samples are an accepted surrogate for bronchial samples [14] that have the advantage of being acquired using minimally invasive techniques. This sampling technique allows for ethical collection from the full range of asthma severity, including youth with mild asthma, and healthy controls.

Samples were collected from fresh tissues and macerated using sterilized plastic tips in $1.5 \mathrm{~mL}$ sterile tubes. Samples in Trizol were frozen immediately at -80 until a later date for batch RNA extraction and processing. Total RNA was extracted using Trizol reagent (Life Technologies) and the resulting lysate was used for affinity RNA purification in silica columns following manufacturer's instructions (Norgen Biotek). RNA quality was assessed by measuring 260/280 absorbance ratio and by integrating proportions of RNA using microchip electrophoresis on Agilent Bioanalyzer 2100 RNA 6000 nanochips (Agilent, Palo Alto CA). Samples with an RNA Integrity Number value greater than five were used for subsequent analysis. Total RNA was subjected to RiboZero ribosomal RNA reduction prior to library preparation using Illumina TrueSeq Stranded Total RNA kit (San Diego, CA) and sequenced on a HiSeq 2500 instrument on two separate 'Rapid' flow cells. This 
generated an average of 41.4 million single-end $100 \mathrm{bp}$ sequencing reads per sample. Sequence data was deposited in the Sequence Read Archive and can be found under the BioProject [SRA: PRJNA255523].

\section{Analyses}

Reads were preprocessed using PRINSEQ-lite 0.20.4 and FastQC 0.10.1 (trimming reads and bases $<25$ PHRED, removing exact duplicates, reads with undetermined bases, and low complexity reads using Dust filter $=30$ ) [15]. We constructed a 'target' genome library containing all bacterial, fungal, and viral sequences from the Human Microbiome Project Reference Database (http://www.hmpdacc.org/reference_genomes/reference genomes.php) using the PathoLib module from PathoScope 2.0 [16]. We aligned reads to these libraries using the Bowtie 2 algorithm [17], and then filtered any reads that also aligned to the human genome (hg19) as implemented in PathoMap (--very-sensitive-local -k 100 -scoremin L,20,1.0). In these samples, an average of 1.8 million reads (9.1\%; range: $4.8 \%-16.72 \%)$ per sample aligned to the target libraries before filtering the human genome. We then applied PathoScope 2.0 - specifically the PathoID module - to characterize the microbial communities in each patient.

Exploratory analysis and differential species abundance testing were performed in R 3.1.2 and Bioconductor 3.0 $[18,19]$ using packages xlsx 0.5.7, gtools 3.4.1, CHNOSZ 1.0.3.1, plyr 1.8.1, ggplot2 1.0.0, reshape2 1.4.1, gplots 2.16.0, Phyloseq 1.10.0, and DESeq2 1.6.3 [20-28]. Briefly, various indices (Observed, Chao1, Shannon, Simpson) were obtained using the plot_richness function of the PhyloSeq package and beta diversity was obtained using the $R$ base package $[19,26]$. Numbers of mapped reads were normalized across all samples using the variance stabilizing transformation method [27, 28]. Relative differences between groups were tested using a Wald test (with Cook's distance correction for outliers) and adjusted by applying the Benjamini-Hochberg method to correct for multiple hypotheses testing at alpha $=0.05[29,30]$. Taxa whose base number of normalized reads was less than 50 were not considered. Principal coordinate analysis (PCoA) was performed on a Jensen-Shannon distance matrix derived from read counts aggregated by genus as estimated in PathoScope.

For gene differential expression analysis, we aligned the dataset to the human genome using TopHat v2.0.6 [31] and estimated the expressed gene abundance using Cufflinks v2.1.1 [32] represented as fragments per kilobase of exon per million fragments mapped (under default parameters). Since $M$. catarrhalis was detected with high proportion of mapped reads in 5/8 asthma samples while with low proportion of mapped reads in all of the control samples, we further evaluated the host response gene expression signature of $M$. catarrhalis in these samples. In a previous study, a list of differential expressed genes (77 genes) was identified in the respiratory tract epithelial cells in response to adherent $M$. catarrhalis BBH18 [33]. We applied an adaptive Bayesian factor analysis approach as implemented in the ASSIGN toolkit from Bioconductor [34]. We estimated the strength of $M$. catarrhalis host gene expression signature onto the samples in our dataset to determine whether this signature is present in the tissue samples of asthma and control samples [16]. For this analysis we used the following parameters: [adaptive_B $=$ TRUE, adaptive_S $=$ TRUE, mixture_beta $=$ TRUE, p_beta $=0.001$, iter $=2000$, burn_in $=1000$, theta $0=0.05$, theta1 $=0.9]$.

\section{Results and discussion}

To our knowledge, this is the first study reporting the use of shotgun RNA sequencing for microbial identification and differential host gene expression. Throughout this study we refer to microbial composition as the combined effect of the presence of a certain microbe and its gene expression. The advantage of this strategy is that measures of relative abundance are related to the actual activity or expression of a microbe at a given point in time instead of to the census number of a microbe. Additionally, it allows for the interrogation of the host transcriptomes or specific gene signatures from the same sequence dataset. Franzosa et al. showed that metagenomic and metatranscriptomic genes and/or species abundance do not necessarily correlate [35], meaning that species' relative abundances reported in this study represent actual activity or expression of microbes, and might not correlate to relative abundance from shotgun DNA experiments.

\section{Asthma microbial communities are less diverse than controls}

We performed analyses of alpha and beta diversity to assess species richness and evenness within and among samples (Fig. 1 and b). We obtained estimates of various indices to characterize the richness and heterogeneity of the samples partitioned by asthma and control samples (Observed, Chao1, Shannon, Simpson). Observed and Chao1 are measures of species richness (number of species); the latter including a correction for unobserved species [36, 37]. In turn, Shannon and Simpson incorporate relative species abundance and thus represent Evenness or Heterogeneity [38]. We observed that asthma samples have more species (richer) compared to control individuals (Fig. 1a; Observed and Chao1). However, measures that explicitly model Evenness (Shannon and Simpson indices) suggest that asthmatic samples are dominated by fewer species ( 5 of 8 cases 


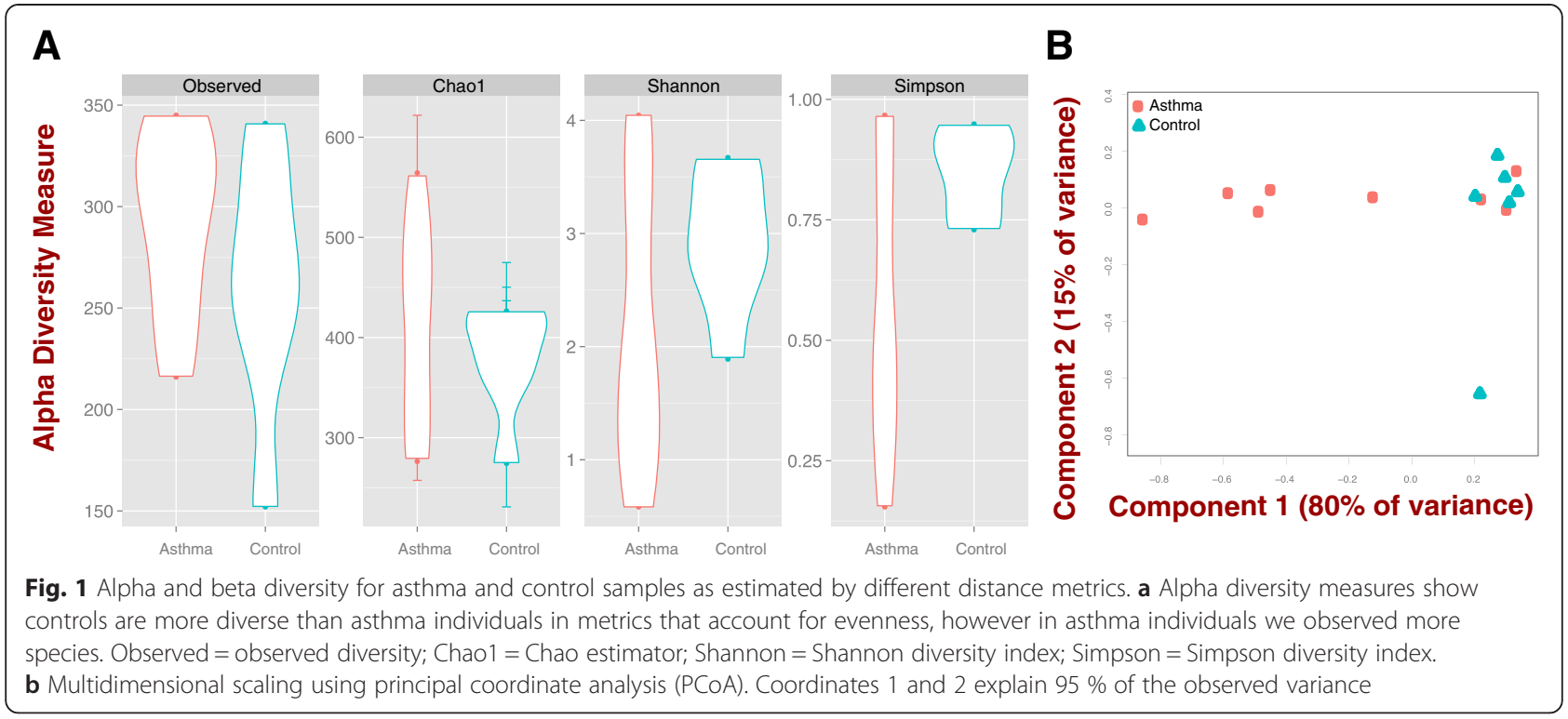

dominated by Moraxella catarrhalis; Fig. 1a; Fig. 2) and are thus less diverse than controls.

Decreased microbial diversity have also been observed in other human diseases [39-41], although increased diversity in diseased patients has also been noted [42]. In asthma studies, bacterial diversity, as surveyed by $16 \mathrm{~S}$ rRNA amplicon sequencing and 16S microarray typing, exhibits an opposite trend to bronchial and induced sputum samples, i.e., asthma samples are more diverse than controls [43, 44]. In addition, other studies have not detected significant differences among asthma samples and controls $[9,45]$. The discordance between our results and previous studies might arise from two sources. We used shotgun RNA sequencing instead of marker-based approaches. The shotgun approach is more comprehensive (virus, bacteria, fungi); thus, it is likely that we sampled the microbiome more extensively. In addition, our estimates might not be directly comparable as we measure abundance as a composite of the product of microbial gene expression and census numbers, i.e., we sampled species that were expressing genes vs. sampling species that were present. Secondly, we sampled a surrogate of bronchial samples, i.e., the nasal cavity of children and adolescents (Table 1), and other studies have directly sampled the lower respiratory tract of adults and children.

Regarding among-sample relatedness, we observe that the five samples where $M$. catarrhalis is relatively more abundant tend to differentiate from controls (PCoA; $95 \%$ of variance), while asthma samples with low levels of $M$. catarrhalis tend to cluster with controls (Fig. 1b). Interestingly, the latter three samples also exhibit the lowest proportion of $M$. catarrhalis, and two of them exhibit no host response to $M$. catarrhalis-associated genes (below; Fig. 4; P001 and P005). Recently, Goleva et al. found no differences either in diversity or composition in patients with corticoid-sensitive or resistant phenotypes compared to controls in samples without $M$. catarrhalis [45]. This suggests that asthma microbiomes where $M$. catarrhalis is not detected resemble those of control individuals, however we do not discard the possibility that another unidentified microbe is driving this apparent similarity.

\section{Microbial identification and relative abundance in asthma and control communities}

The resulting composition differed significantly between the cases and controls at the species level, with 5 of the 8 cases showing high (more than $50 \%$ of mapped reads) prevalence of the bacterial species $M$. catarrhalis (Fig. 2; check Additional File 1 for raw counts). Other abundant species in asthma samples were Corynebacterium accolens, and C. tuberculostearicum. However, these were also found in high abundance in control samples (Fig. 2). Corynebacterium spp. have been detected in sinus nasal studies of healthy individuals as well as in cases of rhinitis and rhinosinusitis, where their prevalence nears $100 \%$ and their abundance is relatively high [46-48].

When we formally test for differential relative abundance, we observed a $\log _{2}$-based effect size of 3.8 for $M$. catarrhalis, i.e., this species is on average 14 times more abundant in children with asthma than controls (Fig. 3a). These findings build on previous metagenomic surveys using 16S rRNA, which found increased proportions of Proteobacteria in cases but not controls, speculating that this could be explained by Moraxella spp. and Hemophilus spp. [9]. M. catarrhalis is a pathogen associated with pneumonia in early childhood [49], and airway colonization shortly after birth with $M$. catarrhalis, along with $H$. influenzae, and S. pneumonia, is associated with later asthma 


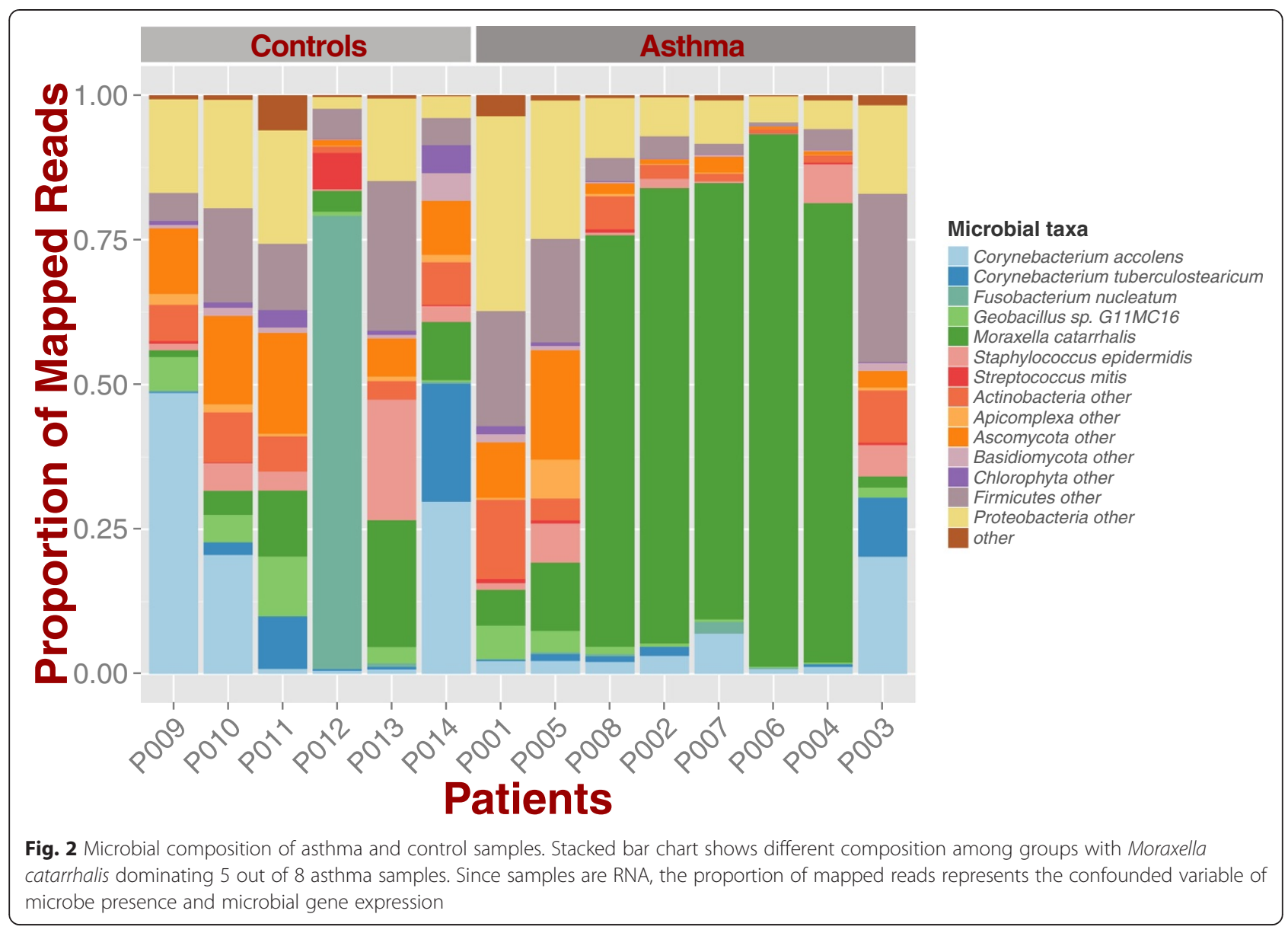

development [5] and with wheezy episodes in young children [50].

We also detected Escherichia and Psychrobacter (Family: Moraxellaceae) to be significantly more abundant in asthma samples than controls, both members of the human microbiome [51, 52]; yet in low quantities (Fig. 3b; 65 and 55 reads, respectively). While we detected H. influenzae, Streptococcus spp., and Staphylococcus spp. in asthma samples, their abundance was not significantly different between asthma and control samples (p-value > 0.05; check Additional File 2 for R code). We also found Anaerococcus prevotii, member of the normal microbiome of the skin, oral cavity and the gut, to be relatively less abundant in asthma samples (Fig. 3a-b; 57 reads on average). These findings, i.e., more Proteobacteria and less Firmicutes in asthma, are in agreement with other reports $[9,44]$.

\section{Host gene expression validates microbial community profiling}

While identifying pathogenic species of a distinct airway microbiome in cases but not in controls is suggestive evidence to implicate an agent for disease, we wanted to further validate this conclusion by examining the host response to M. catarrhalis. Because our starting nucleic acid material is RNA from human epithelial cells, the majority of the sequencing reads are of human origin ( $>95 \%$ mapping to human genome; $75 \%$ mapping to human transcriptome). Thus, we can capitalize on these data to examine host response gene expression through these transcriptomic data. We obtained a set of 77 genes that were previously associated with the immune response to $M$. catarrhalis infection in respiratory tract epithelial cells [33] of which 32 gene names were found in our dataset. We fit this $M$. catarrhalis host response gene expression signature onto our asthma and control samples (Fig. 4a). None of the controls expressed the $M$. catarrhalis response signature (Fig. 4b). For the eight asthma samples, five exhibited a high $M$. catarrhalis signature strength. These five samples included the samples with the four highest scoring read proportions from PathoScope. Samples with high proportion of $M$. catarrhalis exhibited increased expression of mediators of inflammation (e.g., CCL20; IL1A; IRAK2) and apoptosis (e.g., TNF; C8orf4; Fig. 4a). 


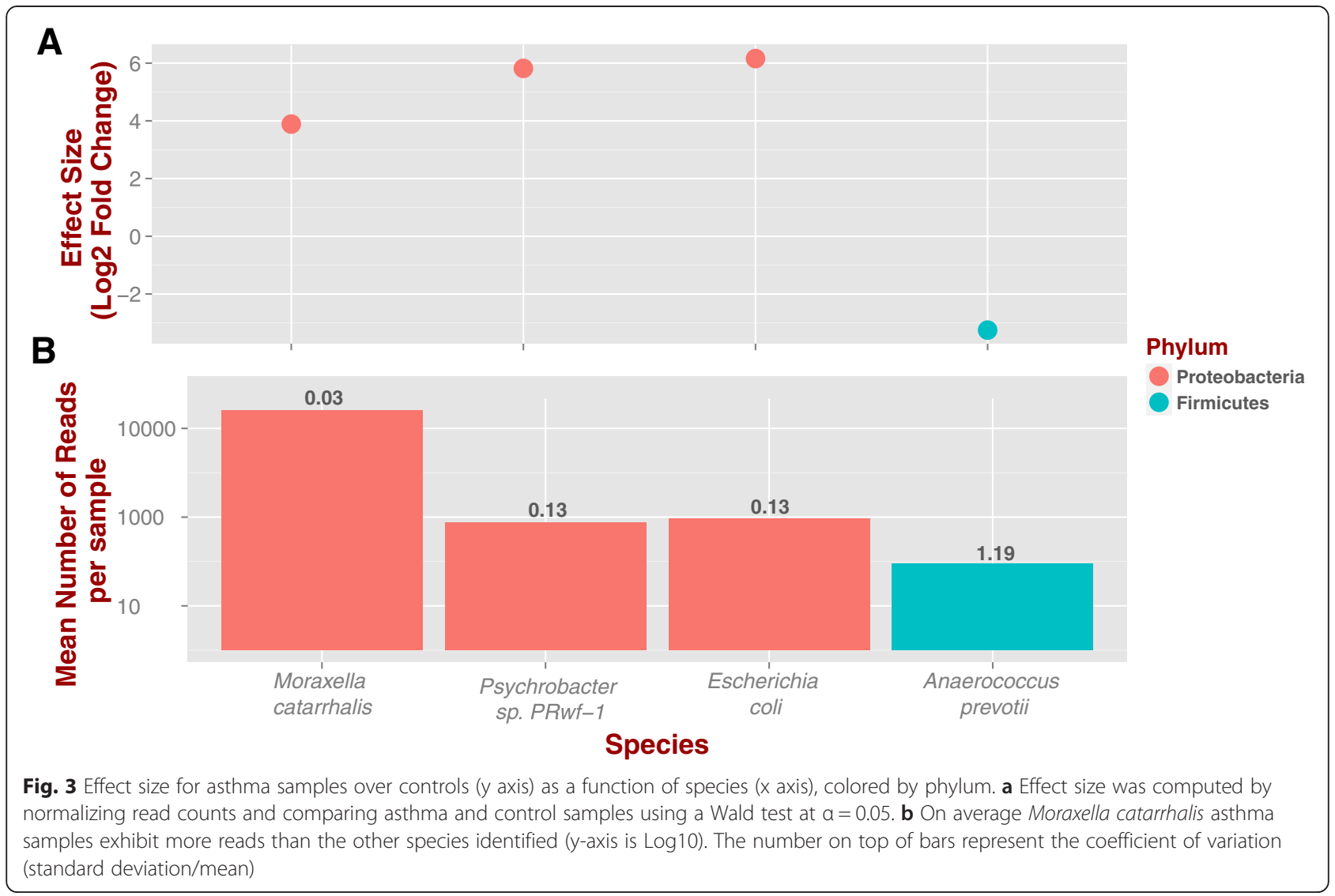

Additionally, signature strength and read map proportions are in concordance for the majority (4 out of 5) of the patient samples (Fig. 4b, c). However, there was one discordant sample with a very low $M$. catarrhalis read proportion (0.02) that scored very high with respect to the gene expression signature (P003 in Fig. 4c). In this sample, we did identify 281 reads from Moraxella spp., indicating that we could still be observing a true host response. Alternatively, this could be a false positive due to lack of specificity in the signature. For instance, in sample P003, we also detected 5249 reads for Corynebacterium, representing $\sim 0.3$ proportion of mapped reads. We did not find a specific signature for Corynebacterium in the literature, however, species in this genus are known to trigger inflammation in the nasal cavity and sinuses, which might explain the strong proinflammatory response $[46,53]$. Altogether, this illustrates the future need for developing a multi-signature approach (i.e., immune response caused by multiple pathogens) that can distinguish between related response signatures. The other two asthma samples (P001 and P005) with no detectable signature for Moraxella showed 304 and $1490 \mathrm{M}$. catarrhalis normalized reads (6 and $12 \%$, respectively). In agreement with our findings, Følsgaard et al. (2014) detected local inflammation markers in nasal mucosal lining fluid samples of neonates after colonization by $M$. catarrhalis, which might lead to the establishment of chronic inflammation [54].

\section{Conclusions}

Our study demonstrates the efficacy of combining microbial identification and host gene signatures for microbial characterization under asymptomatic conditions. In a single shotgun RNA experiment, our integrative approach shows the dominating presence of $M$. catarrhalis in the airways of asthmatic children and the strength of the host immune response against it. This suggests that the airways of asthmatics are chronically inflamed, which may be associated with their ability to respond against opportunistic infections.

While the small sample size of our study, the small number of gene signatures available, and the need for a multi-signature approach render our results as preliminary, we show that our approach simultaneously characterizes the diversity of microbial communities (bacteria, fungi and viruses), and the differential expression of loci from the host in response to an infection. Such a dual approach allows for robust 


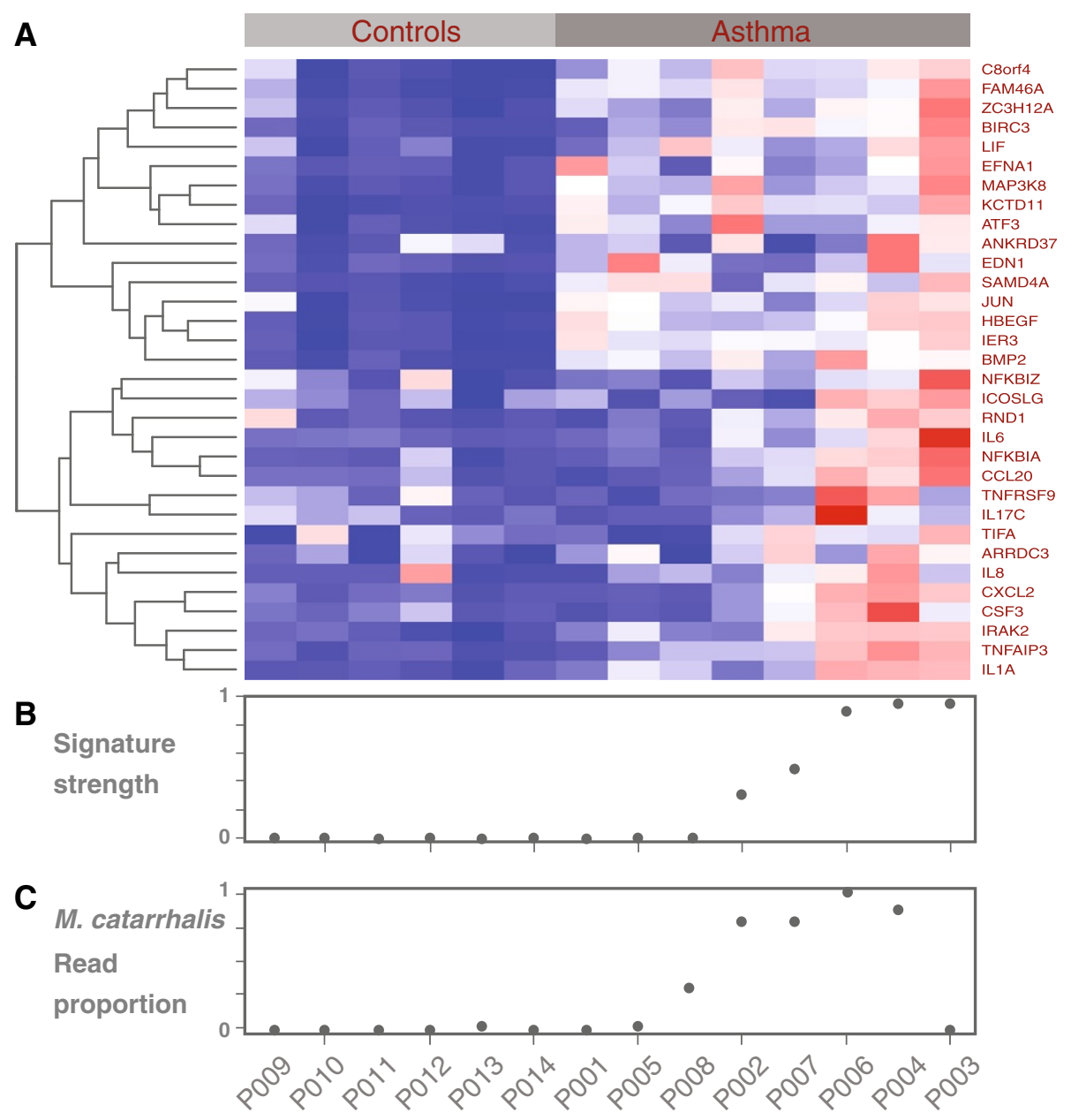

Fig. 4 a Heatmap of Moraxella catarrhalis signature genes distinguishes the asthma samples from the controls. The color scale goes from blue (low expression) to red (high expression). b, c The Moraxella catarrhalis signature strengths are highly concordant with the PathoScope read proportions in control and asthma samples with the exception of sample P003

diagnosis in human health and has a direct and broad applicability in epidemiological, ecological, and medical studies. Future development of multi-species signature statistical approaches along with the availability of more gene signatures will strengthen microbial detection by RNA microbial profiling and host differential gene expression.

\section{Additional files}

Additional file 1: Spreadsheet with taxonomy of species detected and their abundances as read counts. (XLSX $143 \mathrm{~kb}$ )

Additional file 2: OTU and taxonomy tables, metadata, and R code for analysis of differential abundance. (ZIP $32 \mathrm{~kb}$ )

\section{Abbreviations}

rRNA: Ribosomal ribonucleic acid; RIN: RNA integrity number; HTS:

High-throughput sequencing; SRA: Sequence read archive.

\section{Competing interests}

KAC and ECN and WEJ have a combination of ownership of, and employment in, Aperiomics, Inc.

\section{Authors' contributions}

ECN, RJF, WEJ, and KAC conceived and designed the study. ECN, RJF, GL, WEJ, and KAC collected samples and sequence data. ECN and MPL analyzed the data for microbial composition, and YS, WEJ, and SM analyzed the data for host gene signatures. ECN and KAC wrote the manuscript with all authors contributing. All authors read and approved the final manuscript.

\section{Acknowledgements}

ECN would like to thank GW's Computational Biology Institute for funding and GW's Colonial One High-Performance Computing facility for facilitating infrastructure for analyses. ECN was funded by "CONICYT + PAI/CONCURSO NACIONAL APOYO AL RETORNO DE INVESTIGADORES/AS DESDE EL EXTRANJERO, CONVOCATORIA 2014 + FOLIO 82140008". MP-L was funded by a K12 Career Development Program 5 K12 HL119994 award.

\section{Author details}

${ }^{1}$ Computational Biology Institute, George Washington University, Ashburn, VA 20147, USA. ${ }^{2}$ Division of Computational Biomedicine, Boston University School of Medicine, Boston, MA 02118, USA. ${ }^{3}$ Division of Emergency 
Medicine, Children's National Medical Center, Washington, DC 20010, USA. ${ }^{4} \mathrm{CIBIO}-\mathrm{InBIO}$, Centro de Investigação em Biodiversidade e Recursos Genéticos, Universidade do Porto, Campus Agrário de Vairão, Vairão 4485-661, Portugal. ${ }^{5}$ Universidad Andrés Bello, Center for Bioinformatics and Integrative Biology, Facultad de Ciencias Biológicas, Av. República 239, Santiago 8370146, Chile.

\section{Received: 7 October 2014 Accepted: 13 July 2015 Published online: 16 August 2015}

\section{References}

1. Turnbaugh PJ, Ley RE, Hamady M, Fraser-Liggett C, Knight R, Gordon الـ The human microbiome project: exploring the microbial part of ourselves in a changing world. Nature. 2007;449:804.

2. Turnbaugh PJ, Ley RE, Mahowald MA, Magrini V, Mardis ER, Gordon Jl. An obesity-associated gut microbiome with increased capacity for energy harvest. Nature. 2006;444:1027-131.

3. Huang YJ, Charlson ES, Collman RG, Colombini-Hatch S, Martinez FD, Senior RM. The role of the lung microbiome in health and disease. A National Heart, Lung, and Blood Institute workshop report. Am J Respir Crit Care Med. 2013;187:1382-7.

4. Westermann AJ, Gorski SA, Vogel J. Dual RNA-seq of pathogen and host. Nat Rev Microbiol. 2012;10:618-30.

5. Bisgaard H, Hermansen MN, Buchvald F, Loland L, Halkjaer LB, Bønnelykke K, et al. Childhood asthma after bacterial colonization of the airway in neonates. N Engl J Med. 2007;357:1487-95.

6. Vissing $\mathrm{NH}$, Chawes $\mathrm{BL}$, Bisgaard $\mathrm{H}$. Increased risk of pneumonia and bronchiolitis after bacterial colonization of the airways as neonates. Am J Respir Crit Care Med. 2013;188:1246-52.

7. Morris A, Beck JM, Schloss PD, Campbell TB, Crothers K, Curtis JL, et al. Comparison of the respiratory microbiome in healthy nonsmokers and smokers. Am J Respir Crit Care Med. 2013;187:1067-75.

8. Erb-Downward JR, Thompson DL, Han MK, Freeman CM, McCloskey L, Schmidt LA, et al. Analysis of the lung microbiome in the "healthy" smoker and in COPD. PLoS One. 2011;6, e16384.

9. Hilty M, Burke C, Pedro H, Cardenas P, Bush A, Bossley C, et al. Disordered microbial communities in asthmatic airways. PLoS One. 2010;5: e8578.

10. Benton AS, Kumar N, Lerner J, Wiles A, Foerster M, Teach SJ, et al. Airway platelet activation is associated with airway eosinophilic inflammation in asthma. J Investig Med. 2010;58:987.

11. Benton AS, Wang Z, Lerner J, Foerster M, Teach SJ, Freishtat RJ. Overcoming heterogeneity in pediatric asthma: tobacco smoke and asthma characteristics within phenotypic clusters in an African American cohort. J Asthma. 2010;47:728-34

12. Freishtat RJ, Iqbal SF, Pillai DK, Klein CJ, Ryan LM, Benton AS, et al. High prevalence of vitamin D deficiency among inner-city African American youth with asthma in Washington, DC. J Pediatr. 2010;156:948-52.

13. Stemmy EJ, Benton AS, Lerner J, Alcala S, Constant SL, Freishtat RJ. Extracellular cyclophilin levels associate with parameters of asthma in phenotypic clusters. J Asthma. 2011;48:986-93.

14. McDougall CM, Blaylock MG, Douglas JG, Brooker RJ, Helms PJ, Walsh GM. Nasal epithelial cells as surrogates for bronchial epithelial cells in airway inflammation studies. Am J Respir Cell Mol Biol. 2008;39:560-8.

15. Schmieder R, Edwards R. Quality control and preprocessing of metagenomic datasets. Bioinformatics. 2011;27:863-4.

16. Hong C, Manimaran S, Shen Y, Perez-Rogers JF, Byrd AL, Castro-Nallar E, et al. PathoScope 2.0: A complete computational framework for strain identificaion in environmental or clinical sequencing samples. Microbiome 2014, 2.

17. Langmead B, Salzberg SL. Fast gapped-read alignment with Bowtie 2 . Nat Methods. 2012:9:357-9.

18. Gentleman RC, Carey VJ, Bates DM, Bolstad B, Dettling M, Dudoit S, et al. Bioconductor: open software development for computational biology and bioinformatics. Genome Biol. 2004;5:R80.

19. Core RT. R: A language and environment for statistical computing. 2014.

20. Dragulescu AA, Dragulescu MAA, Provide R. Package 'xlsx'. Cell. 2012;9:1.

21. Warnes GR, Bolker B, Bonebakker L, Gentleman R, Huber W, Liaw A, et al. gplots: Various $\mathrm{R}$ programming tools for plotting data. R package version. 2009;2.

22. Dick JM. Calculation of the relative metastabilities of proteins using the CHNOSZ software package. Geochem Trans. 2008;3:10.
23. Wickham H. plyr: Tools for splitting, applying and combining data R package version 01. 2009;9:651.

24. Wickham H. ggplot2. elegant graphics for data analysis. Springer, New York; 2009.

25. Wickham $\mathrm{H}$. Reshaping data with the reshape package. J Stat Softw. 2007;21:1-20.

26. McMurdie PJ, Holmes S. phyloseq: an R package for reproducible interactive analysis and graphics of microbiome census data. PLoS One. 2013;8:e61217.

27. McMurdie PJ, Holmes S. Waste not, want not: Why rarefying microbiome data is inadmissible. PLoS Comput Biol. 2014;10:e1003531.

28. Love Ml, Huber W, Anders S. Moderated estimation of fold change and dispersion for RNA-Seq data with DESeq2. Genome Biology 2014;15:550.

29. Benjamini Y, Hochberg Y. Controlling the false discovery rate: a practical and powerful approach to multiple testing. J R Stat Soc Ser B Methodol. 1995;289-300.

30. Cook RD. Detection of influential observation in linear regression. Technometrics. 1977:15-18.

31. Kim D, Pertea G, Trapnell C, Pimentel H, Kelley R, Salzberg SL. TopHat2: accurate alignment of transcriptomes in the presence of insertions, deletions and gene fusions. Genome Biol. 2013;14:R36.

32. Trapnell C, Roberts A, Goff L, Pertea G, Kim D, Kelley DR, et al. Differential gene and transcript expression analysis of RNA-seq experiments with TopHat and Cufflinks. Nat Protoc. 2012;7:562-78.

33. de Vries SP, Eleveld MJ, Hermans PW, Bootsma HJ. Characterization of the molecular interplay between Moraxella catarrhalis and human respiratory tract epithelial cells. PLoS One. 2013;8, e72193.

34. Shen Y, Rahman M, Piccolo SR, Gusenleitner D, El-Chaar NN, Cheng L, et al. ASSIGN: Context-specific Genomic Profiling of Multiple Heterogeneous Biological Pathways. Bioinformatics 2015;31(11):1745-753.

35. Franzosa EA, Morgan XC, Segata N, Waldron L, Reyes J, Earl AM, et al. Relating the metatranscriptome and metagenome of the human gut. Proc Natl Acad Sci. 2014;111:E2329-38.

36. Chao A. Nonparametric estimation of the number of classes in a population. Scand J Stat. 1984;265-270

37. Colwell RK, Coddington JA. Estimating terrestrial biodiversity through extrapolation. Phil Trans Biol Sci. 1994;345:101-18.

38. Jost L. Partitioning diversity into independent alpha and beta components. Ecology. 2007;88:2427-39.

39. Chang JY, Antonopoulos DA, Kalra A, Tonelli A, Khalife WT, Schmidt TM, et al. Decreased diversity of the fecal microbiome in recurrent Clostridium difficile_-associated diarrhea. J Infect Dis. 2008;197:435-8.

40. Ott S, Schreiber S. Reduced microbial diversity in inflammatory bowel diseases. Gut. 2006;55:1207.

41. Docktor MJ, Paster BJ, Abramowicz S, Ingram J, Wang YE, Correll M, et al. Alterations in diversity of the oral microbiome in pediatric inflammatory bowel disease. Inflamm Bowel Dis. 2012;18:935-42.

42. Liu B, Faller LL, Klitgord N, Mazumdar V, Ghodsi M, Sommer DD, et al. Deep sequencing of the oral microbiome reveals signatures of periodontal disease. PLoS One. 2012;7:e37919.

43. Huang YJ, Nelson CE, Brodie EL, DeSantis TZ, Baek MS, Liu J, et al. Airway microbiota and bronchial hyperresponsiveness in patients with suboptimally controlled asthma. Journal of Allergy and Clinical Immunology 2011, 127:372-381:e373.

44. Marri PR, Stern DA, Wright AL, Billheimer D, Martinez FD: Asthma-associated differences in microbial composition of induced sputum. Journal of Allergy and Clinical Immunology 2013, 131:346-352: e343.

45. Goleva E, Jackson LP, Harris JK, Robertson CE, Sutherland ER, Hall CF, et al. The effects of airway microbiome on corticosteroid responsiveness in asthma. Am J Respir Crit Care Med. 2013;188:1193-201.

46. Abreu NA, Nagalingam NA, Song Y, Roediger FC, Pletcher SD, Goldberg AN, et al. Sinus microbiome diversity depletion and Corynebacterium tuberculostearicum enrichment mediates rhinosinusitis. Sci Transl Med. 2012;4:151ra124.

47. Ramakrishnan VR, Feazel LM, Gitomer SA, Ir D, Robertson CE, Frank DN. The microbiome of the middle meatus in healthy adults. PLoS One. 2013;8:e85507.

48. Bassis CM, Tang AL, Young VB, Pynnonen MA. The nasal cavity microbiota of healthy adults. Microbiome. 2014;2:27

49. Verduin CM, Hol C, Fleer A, van Dijk H, van Belkum A. Moraxella catarrhalis: from emerging to established pathogen. Clin Microbiol Rev. 2002;15:125-44.

50. Bisgaard H, Hermansen MN, Bønnelykke K, Stokholm J, Baty F, Skytt NL, et al. Association of bacteria and viruses with wheezy episodes in young children: prospective birth cohort study. BMJ. 2010;341. 
51. Dewhirst FE, Chen T, Izard J, Paster BJ, Tanner AC, Yu W-H, et al. The human oral microbiome. J Bacteriol. 2010;192:5002-17.

52. Deschaght P, Janssens M, Vaneechoutte M, Wauters G. Psychrobacter isolates of human origin, other than Psychrobacter phenylpyruvicus, are predominantly Psychrobacter faecalis and Psychrobacter pulmonis, with emended description of P. faecalis. Int J Syst Evol Microbiol. 2012;62:671-4.

53. Aurora R, Chatterjee D, Hentzleman J, Prasad G, Sindwani R, Sanford T. Contrasting the microbiomes from healthy volunteers and patients with chronic rhinosinusitis. JAMA Otolaryngol Head Neck Surg. 2013;139:1328-38.

54. Følsgaard NV, Schjørring S, Chawes BL, Rasmussen MA, Krogfelt KA, Brix S,

et al. Pathogenic bacteria colonizing the airways in asymptomatic neonates stimulates topical inflammatory mediator release. Am J Respir Crit Care Med. 2013;187:589-95

\section{Submit your next manuscript to BioMed Central and take full advantage of:}

- Convenient online submission

- Thorough peer review

- No space constraints or color figure charges

- Immediate publication on acceptance

- Inclusion in PubMed, CAS, Scopus and Google Scholar

- Research which is freely available for redistribution 